\title{
Enumeration of bilaterally symmetric 3-noncrossing partitions
}

\author{
Guoce Xinfl and Terence Y. J. Zhang \\ Center for Combinatorics, LPMC-TJKLC, Nankai University, Tianjin 300071, P.R. China
}

\begin{abstract}
Schützenberger's theorem for the ordinary RSK correspondence naturally extends to Chen et. al's correspondence for matchings and partitions. Thus the counting of bilaterally symmetric $k$-noncrossing partitions naturally arises as an analogue for involutions. In obtaining the analogous result for 3-noncrossing partitions, we use a different technique to develop a MAPLE package for 2-dimensional vacillating lattice walk enumeration problems. As an application, we find an interesting relation between two special bilaterally symmetric partitions.
\end{abstract}

Keywords: Partition, tableau, RSK-correspondence, P-recurrence, D-finite.

\section{Introduction}

A partition $P$ of $[n]:=\{1,2, \ldots, n\}$ is a collection of nonempty subsets $\left\{B_{1}, B_{2}, \ldots, B_{k}\right\}$, whose disjoint union is $[n]$. The elements $B_{i}$ are called blocks of $P$. An important special class of partitions are (complete) matchings of $[2 n]$, which are partitions of $[2 n]$ into $n$ two-element blocks. Every partition $P$ of $[n]$ has a graph representation, called partition graph, obtained by identifying vertex $i$ with $(i, 0)$ in the plane for $i=1, \ldots, n$, and drawing an arc connecting $i$ and $j$ above the horizontal axis whenever $i$ and $j$ are (numerically) consecutive in a block of $P$. Such an arc with $i<j$ is called an edge $(i, j)$ of $P$. Let $P^{r e f l}$ denote the partition obtained from $P$ by reflecting in the vertical line $x=(n+1) / 2$. Equivalently, $(i, j)$ is an arc of $P$ if and only if $(n+1-j, n+1-i)$ is an arc of $P^{r e f l}$.

A sequence $\emptyset=\nu^{0}, \nu^{1}, \ldots, \nu^{2 n}=\lambda$ of Young diagrams is called a vacillating tableau of shape $\lambda$ and length $2 n$ if (i) $\nu^{2 i+1}$ is obtained from $\nu^{2 i}$ by doing nothing (i.e., $\nu^{2 i+1}=\nu^{2 i}$ ) or deleting a square, and (ii) $\nu^{2 i}$ is obtained from $\nu^{2 i-1}$ by doing nothing or adding a square.

In what follows, vacillating tableaux are always of shape $\emptyset$ unless specified otherwise. Recently, Chen et al. [4] established a bijection $\phi$ from partitions to vacillating tableaux. Using their bijection, crossings and nestings of a partition are characterized by its corresponding vacillating tableau. When restricting to matchings, the set of images of $\phi$ becomes the set of oscillating tableaux (see Appendix A for definition).

For a vacillating tableau $V$, reading $V$ backward still gives a vacillating tableau, denoted by $V^{\text {rev }}$. Schützenberger's theorem [11] for the ordinary RSK correspondence [6] naturally extends to the bijection $\phi$. The result for partitions is stated as follows.

\footnotetext{
${ }^{\dagger}$ The authors would like to thank the referees for helpful suggestions to improve the presentation, and Christian Krattenthaler and Tom Roby for valuable comments. This work was supported by the 973 Project, the PCSIRT project of the Ministry of Education, the Ministry of Science and Technology and the NSF of China.
}

1365-8050 @ 2008 Discrete Mathematics and Theoretical Computer Science (DMTCS), Nancy, France 
Theorem 1 For any given partition $P$ and vacillating tableau $V, \phi\left(P^{r e f l}\right)=V^{r e v}$ if and only if $\phi(P)=$ $V$.

This result and its analogue for matchings follows trivially from Fomin's growth diagram language. See [5]. The matching case is due to Roby [10] and the partition case is due to Krattenthaler [7].

A vacillating tableau $V$ is said to be palindromic if $V=V^{r e v}$. A partition $P$ of $[n]$ is said to be bilaterally symmetric (bi-symmetric for short) if $P=P^{r e f l}$. Theorem 1 implies that $P$ is bi-symmetric if and only if $V(P)$ is palindromic. The enumeration of bi-symmetric partitions and matchings are not hard, but turns out to be very difficult if we also consider the statistic of crossing number or nesting number. A $k$-subset $\left\{\left(i_{1}, j_{1}\right),\left(i_{2}, j_{2}\right), \ldots,\left(i_{k}, j_{k}\right)\right\}$ of the edge set of a partition $P$ is said to be a $k$-crossing if $i_{1}<i_{2}<\cdots<i_{k}<j_{1}<j_{2}<\cdots<j_{k}$. A $k$-noncrossing partition is a partition with no $k$-crossings. Some nice properties on crossings and nestings of partitions and matchings have been explored in [4]. Here we are interested with the enumeration of these objects.

The number of $k$-noncrossing matchings was enumerated in [4], and the number of bi-symmetric $k$ noncrossing matchings was enumerated in [16]. The number of partitions is well-known to be the Bell number, but a formula for the number of $k$-noncrossing partitions is only known for $k=2$ and $k=3$. See [3]. The number of bi-symmetric partitions was enumerated as the sequence A080107 in [12]. In this abstract we enumerate bi-symmetric $k$-noncrossing partitions for $k=2$ (in Section 5) and $k=3$, which are the same as palindromic vacillating tableaux of height bounded by $k$ for $k=1$ and $k=2$.

Let $\widetilde{C}_{3}(n)$ be the number of bi-symmetric 3 -noncrossing partitions of $[n]$. Our main result is:

Proposition 2 The numbers $\widetilde{C}_{3}(2 n)$ satisfy $\widetilde{C}_{3}(0)=1, \widetilde{C}_{3}(2)=2, \widetilde{C}_{3}(4)=7$, and

$$
\begin{aligned}
27 n(n+2) \widetilde{C}_{3}(2 n)-3\left(7 n^{2}+26 n+27\right) \widetilde{C}_{3}(2 n+2)-\left(7 n^{2}+50 n\right. & +84) \widetilde{C}_{3}(2 n+4) \\
& +(n+5)^{2} \widetilde{C}_{3}(2 n+6)=0 .
\end{aligned}
$$

The numbers $\widetilde{C}_{3}(2 n+1)$ satisfy $\widetilde{C}_{3}(1)=1, \widetilde{C}_{3}(3)=3$, and

$$
9\left(n^{2}+3 n+2\right) \widetilde{C}_{3}(2 n+1)-2\left(5 n^{2}+30 n+43\right) \widetilde{C}_{3}(2 n+3)+(n+4)(n+5) \widetilde{C}_{3}(2 n+5)=0 .
$$

Equivalently, their associated generating functions $\mathcal{G}_{e}(t)=\sum_{n \geq 0} \widetilde{C}_{3}(2 n) t^{n}$ and $\mathcal{G}_{o}(t)=\sum_{n \geq 0} \widetilde{C}_{3}(2 n+$ 1) $t^{n}$ satisfy

$$
\begin{array}{r}
-4-6 t-6 t^{2}+\left(4-12 t-24 t^{2}\right) \mathcal{G}_{e}(t)+\left(5 t-29 t^{2}-57 t^{3}+81 t^{4}\right) \frac{d}{d t} \mathcal{G}_{e}(t) \\
+t^{2}(t-1)(3 t+1)(9 t-1) \frac{d^{2}}{d t^{2}} \mathcal{G}_{e}(t)=0 \\
6+\left(-6+36 t-18 t^{2}\right) \mathcal{G}_{o}(t)+\left(-6 t+50 t^{2}-36 t^{3}\right) \frac{d}{d t} \mathcal{G}_{o}(t)-t^{2}(t-1)(9 t-1) \frac{d^{2}}{d t^{2}} \mathcal{G}_{o}(t)=0
\end{array}
$$

The above result is analogous to that for $C_{3}(n)$, the number of 3-noncrossing partitions of [n], in [3]. By a similar way we represent the generating functions as certain constant terms in Section 2 But the techniques differs thereafter. In proving our result, we develop a MAPLE package in Section 3 that produce $D$-finite equations (see, e.g, [13]) for given constant terms. This applies to a class of two dimensional vacillating lattice walk enumeration problems. Moreover, we find an interesting relation between two types of special bi-symmetric partitions. 


\section{Lattice Path Interpretations and Constant Term Expressions}

In order to prove Proposition 2, we need to introduce the lattice path interpretations. Let $S$ be a subset of $\mathbb{Z}^{k}$. An $S$-vacillating lattice walk of length $n$ is a sequence of lattice points $p_{0}, p_{1}, \ldots, p_{n}$ in $S$ such that i) $p_{2 i+1}=p_{2 i}$ or $p_{2 i+1}=p_{2 i}-e_{j}$ for some unit coordinate vector $e_{j}$; ii) $p_{2 i}=p_{2 i-1}$ or $p_{2 i}=p_{2 i-1}+e_{j}$ for some unit coordinate vector $e_{j}$. We are interested in two subsets of $\mathbb{Z}^{k}: Q_{k}=\mathbb{N}^{k}$ of nonnegative integer lattice points and $W_{k}=\left\{\left(a_{1}, a_{2}, \ldots, a_{k}\right) \in \mathbb{Z}^{k}: a_{1}>a_{2}>\cdots>a_{k} \geq 0\right\}$ of Weyl lattice points. For two lattice points $a$ and $b$ in $W_{k}$ (or $Q_{k}$ ), denote by $w_{k}(a, b, n)$ (or $\left.q_{k}(a, b, n)\right)$ the number of $W_{k}$ (or $Q_{k}$ )-vacillating lattice walks of length $n$ starting at $a$ and ending at $b$. Let $\delta=(k-1, k-2, \ldots, 0)$.

Let $C_{k}(n)$ be the number of $k$-noncrossing partitions of $[n]$. The following consequence of Chen et. al's correspondence $\phi$ is the starting point of the enumeration for 3-noncrossing partitions, as well as for bi-symmetric 3-noncrossing partitions.

Theorem 3 (Chen et al., [4]) The number $C_{k+1}(n)$ equals $w_{k}(\delta, \delta, 2 n)$, i.e., the number of closed $W_{k^{-}}$ vacillating lattice walks of length $2 n$ from $\delta$ to itself.

By the correspondence $\phi, \widetilde{C}_{3}(n)$ is the same as the number of palindromic vacillating tableaux of height bounded by 2 and length $2 n$, and is the same as the number of palindromic $W_{2}$-vacillating lattice walks of length $2 n$ that start and end at $(1,0)$. Since such walks are palindromic, it is sufficient to consider only the first $n$ steps of the lattice walks. We have

$$
\widetilde{C}_{3}(n)=\sum_{b \in W_{2}} w_{2}((1,0), b, n)
$$

Let us introduce the basic idea for solving the problem of determining $C_{3}(n)$, where the $Q_{2}$-vacillating lattice walks starting and ending at $(1,0)$ are considered. The same idea applies to determining $\widetilde{C}_{3}(n)$.

It was shown in [3] by using the reflection principle that

$$
w_{k}(a, b, n)=\sum_{\pi \in \mathfrak{S}_{k}}(-1)^{\pi} q_{k}(\pi(a), b, n)
$$

where $(-1)^{\pi}$ is the sign of $\pi$ and $\pi\left(a_{1}, a_{2}, \ldots, a_{k}\right)=\left(a_{\pi(1)}, a_{\pi(2)}, \ldots, a_{\pi(k)}\right)$. Thus the enumeration of $w_{k}(\delta, \delta, 2 n)$ reduces to that of $q_{k}(a, \delta, 2 n)$. Denote by $a_{i, j}(n)=q_{2}((1,0),(i, j), n)$. Let

and

$$
F_{e}(x, y ; t)=\sum_{i, j, n \geq 0} a_{i, j}(2 n) x^{i} y^{j} t^{2 n}
$$

$$
F_{o}(x, y ; t)=\sum_{i, j, n \geq 0} a_{i, j}(2 n+1) x^{i} y^{j} t^{2 n+1}
$$

be respectively the generating functions of lattice walks of even and odd length. By a step by step construction, one can set up functional equations for $F_{e}(x, y ; t)$ and $F_{o}(x, y ; t)$ and reduces the problem to solving the following functional equation:

$$
K\left(x, y ; t^{2}\right) F_{o}(x, y ; t) / t=x\left(1+x^{-1}+y^{-1}\right)-x^{-1} V_{e}\left(y ; t^{2}\right)-y^{-1} H_{e}\left(x ; t^{2}\right),
$$


where $V_{e}\left(y ; t^{2}\right)$ and $H_{e}\left(x ; t^{2}\right)$ are respectively the generating functions for lattice walks of even length that start at $(1,0)$ and end on the vertical and horizontal axis, and the kernel of the equation $K(x, y ; t)$ is given by

$$
K(x, y ; t)=1-t(1+x+y)\left(1+x^{-1}+y^{-1}\right) .
$$

By the obstinate kernel method of [1, 2], one can finally obtain the generating function $\mathcal{C}(t)$ of $C_{3}(n)$ as

$$
\mathcal{C}(t)=\mathrm{CT}_{x}\left(\left(x^{-2}-x^{2}\right)\left(x^{2}+\left(x^{-2}+x+x^{2}\right) Y+\left(x^{-3}-x^{-1}\right) Y^{2}-x^{-2} Y^{3}\right)\right),
$$

where the operator $\mathrm{CT}_{x}$ extracts the constant term in $x$ of series in $\mathbb{Q}\left[x, x^{-1}\right][[t]]$ and $Y=Y(x ; t)$ is the unique power series in $t$ satisfying $Y=t(1+x+Y)\left(1+\left(1+x^{-1}\right) Y\right)$ given by

$$
Y=\frac{1-\left(x^{-1}+3+x\right) t-\sqrt{\left(1-\left(1+x+x^{-1}\right) t\right)^{2}-4 t}}{2\left(1+x^{-1}\right) t}=(1+x) t+\cdots .
$$

We shall mention that all this is done in the ring $Q\left[x, x^{-1}, y, y^{-1}\right][[t]]$ of formal power series in $t$ with coefficients Laurent polynomial in $x$ and $y$.

This idea works in a similar way for lattice walks starting from a set of points and ending at $(1,0)$. For a set $A$ of points, we denote by $A(x, y)=\sum_{(i, j) \in A} x^{i} y^{j}$ its generating function. Let $C_{3}^{A}(n)$ be the number of $W_{2}$-lattice walks of length $2 n$ starting from points in $A$ and ending at $(1,0)$, and let $\mathcal{C}^{A}(t)$ be the generating function of $C_{3}^{A}(n)$. For instance, $A_{1}(x, y)=x$ corresponds to the point $(1,0)$ and hence $C_{3}^{A_{1}}(n)=C_{3}(n)$ and $\mathcal{C}^{A_{1}}(t)=\mathcal{C}(t)$. For general $A$, with $Y$ as in (7) the result of [3, Section 2.7] for $\mathcal{C}^{A}(t)$ can be summarized as follows.

Proposition 4 For any set $A$ of lattice points in $W_{2}$, we have

$$
\begin{aligned}
\mathcal{C}^{A}(t)=\mathrm{CT}_{x}\left(\left(x^{-2}-x^{2}\right)((x+Y+x Y) A(x, Y)-\right. & \left(x^{-1} Y+Y+x^{-1} Y^{2}\right) A\left(x^{-1} Y, Y\right) \\
& \left.\left.+\left(x^{-1} Y+x^{-1}+x^{-2} Y\right) A\left(x^{-1} Y, x^{-1}\right)\right)\right) .
\end{aligned}
$$

Now, although our lattice walks for $\widetilde{C}_{3}(n)$ always start from $(1,0)$, which is different from that in Proposition 4 , we will still use the formulas for $\mathcal{C}^{A}(t)$ by means of the following two observations:

1) $w_{2}(a, b, 2 n)=w_{2}(b, a, 2 n)$, since $W_{2}$-vacillating lattice walks of even length are still $W_{2}$-vacillating lattice walks if reading backward. Thus by $\left[5\right.$ we obtain $\widetilde{C}_{3}(2 n)=C_{3}^{A_{2}}(2 n)$, where $A_{2}(x, y)=$ $\frac{x}{(1-x)(1-x y)}$ corresponds to the set of all points in $W_{2}$.

2) By the step by step construction we have

$$
w_{2}(a, b, 2 n+1)=w_{2}(a, b, 2 n)+w_{2}(a, b+(1,0), 2 n)+w_{2}(a, b+(0,1), 2 n) .
$$

However, we must take care of the boundary cases. A careful study yields

$$
\sum_{b \in W_{2}} w_{2}(a, b, 2 n+1)=w_{2}(a,(1,0), 2 n)+2 \cdot \sum_{b \in A_{2}^{\prime}} w_{2}(a, b, 2 n)+3 \cdot \sum_{b \in A_{2}^{\prime \prime}} w_{2}(a, b, 2 n),
$$

where $A_{2}^{\prime}(x, y)=x^{2} /(1-x)+x^{2} y /(1-x y)$ and $A_{2}^{\prime \prime}(x, y)=x^{3} y /((1-x)(1-x y))$. 
In summary, with $\mathcal{G}_{e}(t)$ and $\mathcal{G}_{o}(t)$ as stated in Proposition 2 , we have

$$
\begin{aligned}
& \mathcal{G}_{e}(t)=\mathcal{C}^{A_{2}(x, y)}(t), \\
& \mathcal{G}_{o}(t)=\mathcal{C}^{A_{1}(x, y)+2 A_{2}^{\prime}(x, y)+3 A_{2}^{\prime \prime}(x, y)}(t) .
\end{aligned}
$$

Then by Proposition $4, \mathcal{G}_{e}(t)$ and $\mathcal{G}_{o}(t)$ can be represented as certain constant terms. Such constant terms will be systematically dealt with by the MAPLE package developed in Section 3 .

We conclude this section by an interesting result that can be obtained similarly. Let $A_{3}(x, y)=x /(1-$ $x)$ and $A_{4}(x, y)=x /(1-x y)$. Then $A_{3}$ corresponds to the $x$-axis in $W_{2}$ and $A_{4}$ corresponds to the diagonal in $W_{2}$. Denote by $e(n)=C_{3}^{A_{3}}(n)$ and $h(n)=C_{3}^{A_{4}}(n)$. Then $e(n)$ is the number of bisymmetric 3 -noncrossing partitions on $[2 n]$ whose central Young diagram consist of at most one row, and similarly $h(n)$ is the number of bi-symmetric 3-noncrossing partitions on $[2 n]$ whose central Young diagrams consist of two rows of squares of equal length (including $\emptyset$ ).

Proposition 5 For $n \geq 1$, we have $e(n)=2 \cdot h(n)$. Moreover $h(1)=1, h(2)=3$ and

$$
9 n(n+3) h(n)-2\left(5 n^{2}+26 n+30\right) h(n+1)+(n+4)(n+5) h(n+2)=0 .
$$

\section{Determine the Constant Terms by a Maple Package}

In this section we will develop a MAPLE package to find the $D$-finite equation satisfied by $\mathcal{C}^{A}(t)$ from its constant term expression given in Proposition 4 Our proof is based on the idea of Lipshitz [8], but for our particular problem we find a much smaller bound for the degree of the D-finiteness. Moreover, this bound is for a large class of power series and can be carried out by MAPLE. We find it better to work in the field $\mathbb{Q}((x))((t))$ of iterated Laurent series, which is also the field of Laurent series in $t$ with coefficients Laurent series in $x$. See [14, 15] for other applications of this field.

Many objects are easy to describe using

$$
u=\left(x^{-1}+2+x\right)=x^{-1}(1+x)^{2} .
$$

Let

$$
\Delta \equiv \Delta(x, t)=\sqrt{\left(1-\left(1+x+x^{-1}\right) t\right)^{2}-4 t}=\sqrt{(1-(u-1) t)^{2}-4 t} .
$$

Then it is easy to see that $\mathbb{Q}(x, t, \Delta)=\mathbb{Q}(x, t) \oplus \mathbb{Q}(x, t) \Delta$. Since

$$
Y=\frac{1}{2} \frac{x-\left(1+x^{2}+3 x\right) t-x \Delta}{t(1+x)},
$$

$\mathcal{C}^{A}(t)$ can be written as

$$
\mathcal{C}^{A}(t)=\underset{x}{\mathrm{CT}} T_{0}+\underset{x}{\mathrm{CT}} T_{1} \Delta
$$

for some $T_{0}, T_{1} \in \mathbb{Q}(x, t)$. In our study, the series $A(x, y)$ is always of the form $P(x, y) /((1-x)(1-x y))$ for some polynomial $P(x, y)$. Consequently the rational functions $T_{0}$ and $T_{1}$ may have $x, 1+x, D_{1}, D_{2}$, and $D_{3}$ (but no more) as denominators that are not free of $x$, where

$$
D_{1}=-2 t-(5 t-1) x-2 t x^{2}=x(1-(2 u+1) t)
$$




$$
\begin{gathered}
D_{2}=-t-2 t x-(3 t-1) x^{2}-2 t x^{3}-t x^{4}=x^{2}\left(1-(u-1)^{2} t\right), \\
D_{3}=t^{2}+\left(2 t^{2}-2 t\right) x+\left(3 t^{2}-6 t+1\right) x^{2}+\left(2 t^{2}-2 t\right) x^{3}+t^{2} x^{4}=\Delta^{2} x^{2}=x^{2}\left((1-(u-1) t)^{2}-4 t\right) .
\end{gathered}
$$

Of course one can write everything in terms of $Y$, but use of $\Delta$ may significantly simplify the proof because the derivatives of $\Delta$ have simple expressions. We have

$$
\begin{aligned}
\frac{\partial}{\partial x} \Delta(x, t) & =\frac{t^{2} x^{4}+\left(t^{2}-t\right) x^{3}-\left(t^{2}-t\right) x-t^{2}}{x D_{3}} \Delta, \\
\frac{\partial}{\partial t} \Delta(x, t) & =\frac{t x^{4}+(2 t-1) x^{3}+(3 t-3) x^{2}+(2 t-1) x+t}{D_{3}} \Delta .
\end{aligned}
$$

Let $\mathcal{L}$ be the (finite) $\mathbb{Q}(t)$-linear span of

$$
\left\{\mathrm{CT}_{x} \frac{L(x, t) \Delta}{(1+x)^{p} D_{1}^{q} D_{2}^{r} D_{3}^{s}} \mid(p, q, r, s) \in \mathbb{Z}^{4}, L(x, t) \text { is a Laurent polynomial }\right\} .
$$

We shall devote ourselves to prove the following result.

Proposition 6 The linear span $\mathcal{L}$ is of dimension at most 3. More precisely, for any given $L(x, t), p, q, r$ and $s$, there exists a procedure to find rational functions $R(t), P(t), Q(t) \in \mathbb{Q}(t)$ such that

$$
\mathrm{CT}_{x} \frac{L(x, t) \Delta}{(1+x)^{p} D_{1}^{q} D_{2}^{r} D_{3}^{s}}=R(t)+\mathrm{CT}_{x}(P(t)+Q(t) x) \Delta .
$$

It is clear that $\mathcal{L}$ is closed under taking derivatives with respect to $t$, so we have the following consequence. See [13] for the definition of $D$-finite.

Corollary 7 Every element in $\mathcal{L}$ is $D$-finite of order at most 2.

The basic idea for proving Proposition 6 is to use the well-known formula

$$
\mathrm{CT}_{x} x \frac{\partial}{\partial x} F(x, t)=0, \text { for all } F(x, t) \in \mathbb{Q}((x))((t))
$$

to reduce elements of $\mathcal{L}$ into simple form. We need the following lemma.

Lemma 8 a) For all $k \in \mathbb{Z}$, we have

b)

$$
\begin{gathered}
\mathrm{CT}_{x}\left(x^{k}-x^{-k}\right) \Delta=0 \\
\mathrm{CT}_{x}\left(x^{k}-x^{-k}\right) \frac{x \Delta}{D_{1}}=0, \\
\mathrm{CT}_{x}\left(x^{k}-x^{-k}\right) \frac{x^{2} \Delta}{D_{2}}=0 . \\
\mathrm{CT}_{x} \frac{1-x}{1+x} \Delta=1-t . \\
\mathrm{CT}_{x} \frac{1-x}{1+x} \frac{x \Delta}{D_{1}}=1 . \\
\mathrm{CT}_{x} \frac{1-x}{1+x} \frac{x^{2} \Delta}{D_{2}}=1 . \\
\mathrm{CT}_{x}(1-x)(1-3 x t) \Delta=(1-t)^{2} .
\end{gathered}
$$$$
\text { c) }
$$ 
Proof: For brevity and similarity, we only prove ( $\mathrm{P} 4 \mathrm{P} 7 \mid \mathrm{P} 8)$. Using the easy fact

$$
\mathrm{CT}_{x} F(x, t)=\underset{x}{\mathrm{CT}} F\left(x^{-1}, t\right), \text { if } F(x, t) \in \mathbb{Q}\left[x, x^{-1}\right][[t]],
$$

we can prove ( $\mathrm{P} 4 \mathrm{f}$ by letting $F(x, t)=\Delta(x, t) /\left(D_{2} / x^{2}\right)$ and observing $F(x, t)=F\left(x^{-1}, t\right)$.

For part b), we use Jacobi's change of variable formula [15] in the one variable case:

Theorem 9 (Jacobi's Residue Formula) Let $y=f(x) \in \mathbb{C}((x))$ be a Laurent series and let be the integer such that $f(x) / x^{b}$ is a formal power series with nonzero constant term. Then for any formal series $G(y)$ such that the composition $G(f(x))$ is a Laurent series, we have

$$
\mathrm{CT}_{x} G(f(x)) \frac{x}{f} \frac{\partial f}{\partial x}=b \underset{y}{\mathrm{CT}} G(y) \text {. }
$$

We make the change of variable by $f(x)=u=x^{-1}+2+x=x^{-1}(1+x)^{2}$ with $b=-1$. It is worth mentioning that the $y$ on the right-hand side of (14) is understood the same as $x^{-1}$ (or "very large"). For instance, $G(y)=1 /(1-y)$ should be expanded as $1 /(-y(1-1 / y))=\sum_{n \geq 0}-y^{-1-n}$. See [15] for detailed explanation. Though this understanding is not used in our calculation since $G(y)$ will be taken as Laurent polynomials, it is crucial if we make a more natural change of variable by $f(x)=x^{-1}+1+x$.

Direct calculation shows that

$$
\frac{x}{u} \frac{\partial u}{\partial x}=\frac{x^{2}}{(1+x)^{2}}\left(1-x^{-2}\right)=-\frac{1-x}{1+x} .
$$

Thus Jacobi's Residue Formula gives us the following equality

$$
\mathrm{CT}_{x} G(u(x)) \frac{1-x}{1+x}=\mathrm{CT}_{u} G(u) .
$$

Noticing that $G(u)=\frac{\sqrt{(1-(u-1) t)^{2}-4 t}}{1-(u-1)^{2} t}$ is a power series in both $u$ and $t$, we have

$$
\mathrm{CT}_{x} \frac{1-x}{1+x} \frac{\Delta}{D_{2} / x^{2}}=\mathrm{CT}_{u} \frac{\sqrt{(1-(u-1) t)^{2}-4 t}}{1-(u-1)^{2} t}=\frac{\sqrt{(1+t)^{2}-4 t}}{1-t}=1 .
$$

c) By $(\overline{\mathrm{P} 1})$ and $(\overline{\mathrm{P} 2})$, the following easily verified equation (from later calculation)

$$
\begin{aligned}
\frac{\partial}{\partial t} \frac{(1-x)(1-3 t x)}{(1-t)^{2}} \Delta=\frac{1+3 t}{(t-1)^{3}}\left(x-x^{-1}\right) \Delta & \\
& -x \frac{\partial}{\partial x} \frac{4 t x^{3}+\left(3 t^{2}-7 t\right) x^{2}+\left(3 t^{2}-4 t-3\right) x+3 t^{2}+t}{2(t-1)^{3} t x} \Delta
\end{aligned}
$$

shows that $\mathrm{CT}_{x}(1-x)(1-3 x t)(1-t)^{-2} \Delta$ is a constant. Equation $(\mathrm{P} 8)$ thus follows by checking the $t=0$ case.

Proof of Proposition 6: Clearly we can assume that $p, q, r, s \geq 1$. Let $\operatorname{deg}_{x} D$ be the degree of $D$ in $x$. By classical results for partial fraction decompositions, we have the unique decomposition

$$
\frac{L(x, t)}{(1+x)^{p} D_{1}^{q} D_{2}^{r} D_{3}^{s}}=l(x, t)+\sum_{i=1}^{p} \frac{P_{i}}{D_{0}^{i}}+\sum_{i=1}^{q} \frac{Q_{i}(x, t)}{D_{1}^{i}}+\sum_{i=1}^{r} \frac{R_{i}(x, t)}{D_{2}^{i}}+\sum_{i=1}^{s} \frac{S_{i}(x, t)}{D_{3}^{i}}
$$


where $l(x, t)$ is a Laurent polynomial in $x, D_{0}=1+x, P_{i} \in \mathbb{R}, \operatorname{deg}_{x} Q_{i}(x, t)<\operatorname{deg}_{x} D_{1}, \operatorname{deg}_{x} R_{i}(x, t)<$ $\operatorname{deg}_{x} D_{2}$ and $\operatorname{deg}_{x} S_{i}(x, t)<\operatorname{deg}_{x} D_{3}$ for all $i$.

When we say to get rid of $D_{i}^{j}$, we mean to get rid of the term containing $D_{i}^{j}$ as denominator without producing new denominators in the partial fraction decomposition.

Step 1: Successively get rid of $D_{2}^{r}, D_{2}^{r-1}, \ldots, D_{2}^{2}$, and similarly for $D_{1}$ and $D_{0}$. The process works for any irreducible polynomial $D=D(x, t)$ that is coprime to $x$ and $D_{3}$. Noticing

$$
D_{3}=x^{2} \Delta^{2} \Rightarrow \frac{\partial \Delta}{\partial x}=-\frac{\Delta}{x}+\frac{1}{2} \frac{\Delta}{D_{3}} \frac{\partial D_{3}}{\partial x},
$$

we can eliminate $N \Delta / D^{r}$ for $r \geq 2$ and $\operatorname{deg}_{x} N<\operatorname{deg}_{x} D$ by subtracting the following:

$$
0=\underset{x}{\mathrm{CT}} x \frac{\partial}{\partial x} \frac{S \Delta}{D^{r-1}}=\mathrm{CT}_{x}\left(\frac{x \Delta}{D^{r-1}} \frac{\partial S}{\partial x}-\frac{S \Delta}{D^{r-1}}+\frac{1}{2} \frac{x S \Delta}{D^{r-1} D_{3}} \frac{\partial D_{3}}{\partial x}+\frac{(1-r) x S \Delta}{D^{r}} \frac{\partial D}{\partial x}\right) .
$$

Here $S$ is an appropriately chosen polynomial in $x$. Clearly we need to choose $S$ such that $D$ divides $(1-r) \frac{\partial D}{\partial x} x S-N$. Since $D$ is irreducible and coprime to $x$, it is coprime to $x \frac{\partial D}{\partial x}$. Therefore we can find polynomials $\alpha$ and $\beta$ in $x$ (by the Euclidean algorithm) such that

$$
\alpha D+\beta x \frac{\partial D}{\partial x}=1 \text {. }
$$

Now choose

$$
S=N \beta /(1-r) \Rightarrow(1-r) \frac{\partial D}{\partial x} x S-N=-\alpha N D .
$$

Step 2: Get rid of $D_{0}$ by using $(\overline{\mathrm{P} 5})$, which can be rewritten as

$$
\mathrm{CT}_{x}(1+x)^{-1} \Delta=(1-t) / 2+1 / 2 \mathrm{CT}_{x} \Delta .
$$

Get rid of $D_{1}$ by subtracting a linear combination of the following two constant terms.

$$
\begin{gathered}
\mathrm{CT}_{x} \frac{1-x}{1+x} \frac{x \Delta}{D_{1}}-1=\mathrm{CT}_{x}\left(-\frac{\Delta}{t-1}-\frac{4 t+(5 t-1) x}{(t-1) D_{1}} \Delta\right)=0, \\
\mathrm{CT}_{x} \frac{\partial}{\partial x}\left(\ln (1-Y / x)-\frac{1}{2} \ln \left(D_{1} / x\right)\right)=\underset{x}{\mathrm{CT}}\left(-\frac{1}{4}+\frac{\Delta}{4(t-1)}+\frac{x \Delta}{D_{1}}-\right. \\
\left.\frac{t^{2}+\left(t^{2}-2 t\right) x+\left(t^{2}-t-1\right) x^{2}+t x^{3}}{2(t-1) D_{3}} \Delta\right)=0 .
\end{gathered}
$$

Step 3: Eliminate $x^{k} \Delta / D_{2}$ for $k=1,2,3$ using the following three constant terms.

$$
\begin{aligned}
\mathrm{CT}_{x} \frac{\left(x^{3}-x\right) \Delta}{D_{2}} & =0 \quad(\text { by }(\mathrm{P} 4) \text { with } k=1) \\
\mathrm{CT} \frac{1-x}{1+x} \frac{x^{2} \Delta}{D_{2}}-1 & =\mathrm{CT}_{x}\left(-\frac{\Delta}{t-1}-\frac{2 t+4 t x+(3 t-1) x^{2}+2 t x^{3}}{(t-1) D_{2}} \Delta\right)=0 \\
\mathrm{CT}_{x} x \frac{\partial}{\partial x}\left(\ln (1-x Y)-\frac{1}{2} \ln \left(D_{2} / x^{2}\right)\right)=\mathrm{CT}_{x}\left(\frac{3}{4}+\frac{\Delta}{4(t-1)}-\frac{\Delta x^{2}}{D_{2}}\right. & \left.-\frac{t^{2}+\left(t^{2}-2 t\right) x+\left(3 t^{2}-5 t+1\right) x^{2}+t x^{3}}{2(t-1) D_{3}} \Delta\right)=0
\end{aligned}
$$


Step 4: Get rid of $D_{3}$ similarly as in Step 1, including the case $r=1$. By collecting terms in 15) (with $D=D_{3}$ ), with $\alpha$ and $\beta$ in $\left(16\right.$, we can eliminate $N \Delta / D_{3}^{r}$ by choosing

$$
S=N \beta /(3 / 2-r) \Rightarrow\left(\frac{3}{2}-r\right) \frac{\partial D_{3}}{\partial x} x S-N=-\alpha N D_{3}
$$

Step 5: First, eliminate the $x^{s} \Delta$ terms for $s<0$ by ( $(\overline{\mathrm{P} 2})$. Then eliminate the $x^{s} \Delta$ terms for $s=$ $k, k-1, \ldots, 3$, where $k=\max \left\{\operatorname{deg}_{x} l(x, t), \operatorname{deg}_{x} l\left(x^{-1}, t\right)\right\}$, one by one by the formulas

$$
\begin{aligned}
x \frac{\partial}{\partial x} x^{1+i} \Delta^{3} & =\left((4+i) t^{2} x^{i+3}+b x^{i+2}+b^{\prime} x^{i+1}+b^{\prime \prime} x^{i}\right) \cdot \Delta, \text { for } i \geq 1, \\
x \frac{\partial}{\partial x} x \Delta^{3} & =\left(4 t^{2} x^{3}+\left(5 t^{2}-5 t\right) x^{2}+\left(3 t^{2}+1-6 t\right) x-t^{2}+t-2 \frac{t^{2}}{x}\right) \Delta,
\end{aligned}
$$

where the $b$ 's are independent of $x$, by $[\overline{\mathrm{P} 8}]$ for $s=2$, and by $[\overline{\mathrm{P} 2}]$ for $s=-1$.

Step 6: Eliminate $\Delta / D_{2}$ and hence all terms containing $D_{2}$ (see Step 3) by using the following equality, whose discovery will be discussed in Section 4.

$$
\begin{aligned}
\mathrm{CT}_{x}\left(\frac{9}{32} \frac{1}{t^{2}(9 t-1)}\right. & \left(-1+8 t+55 t^{2}-440 t^{3}+861 t^{4}-528 t^{5}+45 t^{6}\right) \\
+ & \left(\frac{9}{32} \frac{1}{t^{2}(9 t-1)}\left(1-5 t-74 t^{2}+210 t^{3}-87 t^{4}-45 t^{5}\right)\right. \\
+ & \left.\frac{9}{32} \frac{1}{t^{2}(9 t-1)}\left(-4+8 t+240 t^{2}-552 t^{3}+180 t^{4}\right) t x\right) \Delta \\
& \left.-\frac{9}{8} \frac{\Delta\left(1+5 t-21 t^{2}+15 t^{3}\right)}{t D_{2}}\right)=0 .
\end{aligned}
$$

Denote by $E(t)$ the the left-hand side of the above equation. To show that $E(t)=0$, we first show that $E(t)$ satisfies a $D$-finite equation. The method is typical.

Using Steps 1-5, we can rewrite

$$
\frac{d^{i}}{d t^{i}} E(t)=\widetilde{R}_{i}(t)+\mathrm{CT}_{x}\left(\widetilde{P}_{i}(t)+\widetilde{Q}_{i}(t) x\right) \Delta+\mathrm{CT}_{x} \widetilde{S}_{i}(t) \Delta / D_{2}, \quad i=0,1,2,3,4 .
$$

By solving the system of equations

$$
\left\{\begin{array}{l}
a \widetilde{R}_{0}(t)+b \widetilde{R}_{1}(t)+c \widetilde{R}_{2}(t)+d \widetilde{R}_{3}(t)+e \widetilde{R}_{4}(t)=0 \\
a \widetilde{P}_{0}(t)+b \widetilde{P}_{1}(t)+c \widetilde{P}_{2}(t)+d \widetilde{P}_{3}(t)+e \widetilde{P}_{4}(t)=0 \\
a \widetilde{Q}_{0}(t)+b \widetilde{Q}_{1}(t)+c \widetilde{Q}_{2}(t)+d \widetilde{Q}_{3}(t)+e \widetilde{Q}_{4}(t)=0 \\
a \widetilde{S}_{0}(t)+b \widetilde{S}_{1}(t)+c \widetilde{S}_{2}(t)+d \widetilde{S}_{3}(t)+e \widetilde{S}_{4}(t)=0
\end{array}\right.
$$

for $a, b, c, d, e$ independent of $x$, we get the nontrivial solution

$$
a=1, b=\frac{2 t+10 t^{2}-42 t^{3}+30 t^{4}}{3+5 t+21 t^{2}-45 t^{3}}, c=d=e=0 .
$$


This implies that $E(t)+b \cdot \frac{d}{d t} E(t)=0$. Solving this differential equation gives

$$
E(t)=C_{0}\left(15 t^{\frac{3}{2}}-21 t^{\frac{1}{2}}+5 t^{-\frac{1}{2}}+t^{-\frac{3}{2}}\right)
$$

for some constant $C_{0}$.

On the other hand, by using MAPLE to expand $E(t)$ as a series in $t$ and then take constant term in $x$, we see that $E(t)$ is actually a power series in $t$ with $E(0)=0$. It then follows that $C_{0}$ must be 0 and hence $E(t)=0$ as desired.

Once Proposition 6 is established, the differential equations (e.g., (4)) can be proved by MAPLE. The package can be downloaded at http://www.combinatorics.net.cn/homepage/xin/maple/bs3np.txt.

\section{Discussion}

The very general theory in [8] asserts that $\mathcal{C}^{A}(t)$ is $D$-finite if $A(x, y)$ is rational. That is, it satisfies a linear differential equation with polynomial coefficients, or equivalently, $C^{A}(n)$ satisfies a P-recurrence. However the degree of the equations suggested in [8] is usually too large for proving simple P-recurrences as we consider. Note that these recurrences can be easily guessed, using the MAPLE package GFUN. The recurrence for $C_{3}(n)$ was proved by using the Lagrange inversion formula to give a single sum formula and then applying the creative telescoping of [9]. However, the same route is difficult to be applied to our case. The Lagrange inversion formula will give us a complicated double sum.

Actually our MAPLE package can produce the differential equation for $\mathcal{C}^{A}(t)$ for any $A=P(x, y) /((1-$ $x)(1-x y))$ with $P(x, y)$ a polynomial. The whole process will be completed within seconds if $P(x, y)$ is simple. Two curious observations are worth mentioning. We have described how to write $\mathcal{C}^{A}(t)$ as $\mathrm{CT}_{x} T_{0}+\mathrm{CT}_{x} T_{1} \Delta$ for rational $T_{0}$ and $T_{1}$, and Proposition 6 deals with $\mathrm{CT}_{x} T_{1} \Delta$. In practice, we find that i) $T_{0}$ does not contain $D_{2}$ and $D_{3}$ as denominators; ii) using the constant term identity

$$
\mathrm{CT}_{x} \frac{4 t+(5 t-1) x}{2 t+(5 t-1) x+2 t x^{2}}=1 \text {, }
$$

obtained by considering the constant term of $x \frac{\partial}{\partial x} \ln D_{1}$, one sees that $\mathrm{CT}_{x} T_{0}$ is always a rational function in $t$. We do not know why $\mathrm{CT}_{x} T_{0}$ is always rational, since this is not true if we take, e.g., $A(x, y)=$ $x /(1-x)^{2}$ or if we pick out a term from the sum in $(8)$. It is not a problem even if $T_{0}$ has $D_{1}, D_{2}, D_{3}$ as denominators. We can suitably enlarge $\mathcal{L}$ and increase the dimension bound to fit in our package.

In the proof of Proposition 6, only using Steps 1,4,5, one can already give an upper bound for the dimension of $\mathcal{L}$. Theoretically one can prove differential equations like 3 similarly as in Step 6. Such equations, once proved, will reduce the upper bound of the dimension. Equation $(\overline{\mathrm{P} 8})$ is actually obtained when proving the differential equation satisfied by the generating function $\mathcal{C}(t)$ (See [3, Proposition 1]); The equation $E(t)=0$ is obtained when proving $(9)$. Finding small upper bounds for this type of problems may help discovering and proving new formulas, and possibly reducing the upper bound again. This idea may well apply to other situations.

Our contribution is to reduce the upper bound to only 3 . This results in a fast algorithm for 2dimensional vacillating lattice walk enumeration problems. The number 3 should be the actual dimension of $\mathcal{L}$, since otherwise $\mathcal{C}(t)$ must satisfy a lower degree differential equation, which is not suggested by the MAPLE package GFUN. However it seems hard to prove the equality. 


\section{A Appendix: Enumeration of Bi-symmetric Noncrossing Partitions}

In this section we consider the enumeration of bi-symmetric noncrossing partitions. To state our result, we need the following definition: An oscillating tableau (or up-down tableau) of shape $\mu$ and length $n$ is a sequence $\left(\emptyset=\mu^{0}, \mu^{1}, \ldots, \mu^{n}=\mu\right)$ of partitions such that for all $1 \leq i \leq n-1$, the diagram of $\mu^{i}$ is obtained from $\mu^{i-1}$ by either adding or removing one square.

Proposition 10 There is a bijection between the set of palindromic oscillating tableaux of length $2 n$ and height bounded by 1 and the set of palindromic vacillating tableaux of length $2 n$ and height bounded by 1. Moreover, both of them are enumerated by $\left(\begin{array}{c}n \\ \lfloor n / 2\rfloor\end{array}\right)$.

To construct the bijection, it is convenient to introduce an intermediate set $\mathcal{W}(n)$ of all 01 words of length $n$ with no initial segments containing more 0's than 1's. It is well-known that $|\mathcal{W}(n)|=\left(\begin{array}{c}n \\ \lfloor n / 2\rfloor\end{array}\right)$. For any 01 word $w$ of length $n$, define

$\operatorname{odd}(w, s)=\mid\left\{i\right.$ is odd $\left.\mid w_{i}=s, i \in[n]\right\}|, \quad \operatorname{even}(w, s)=|\left\{i\right.$ is even $\left.\mid w_{i}=s, i \in[n]\right\} \mid$, for $s=0,1$.

Then we have the following characterization.

Lemma $11 w \in \mathcal{W}(n) \Leftrightarrow$ for any initial segment $w^{\prime}$ of $w$, even $\left(w^{\prime}, 1\right) \geq \operatorname{odd}\left(w^{\prime}, 0\right)$.

Proof: Let $w^{\prime}$ be the initial segment of $w$ of length $m$. Then we have the natural equality

$$
\operatorname{even}\left(w^{\prime}, 1\right)+\operatorname{even}\left(w^{\prime}, 0\right)+\chi(m \text { is odd })=\operatorname{odd}\left(w^{\prime}, 0\right)+\operatorname{odd}\left(w^{\prime}, 1\right)
$$

where $\chi(S)$ is 1 if the statement $S$ is true and 0 otherwise. On the other hand, by definition $w \in \mathcal{W}(n)$ if and only if for every initial segment $w^{\prime}$ of $w$ we have

$$
\operatorname{even}\left(w^{\prime}, 1\right)+\operatorname{odd}\left(w^{\prime}, 1\right) \geq \operatorname{even}\left(w^{\prime}, 0\right)+\operatorname{odd}\left(w^{\prime}, 0\right) .
$$

Obviously (19) can be replaced with (18) + (19), which is equivalent to even $\left(w^{\prime}, 1\right) \geq \operatorname{odd}\left(w^{\prime}, 0\right)$.

Proof of Proposition 10: Given a palindromic oscillating tableau $O=\left(O_{0}, O_{1}, \ldots, O_{2 n}\right)$ of length $2 n$ and height bounded by 1 , we have a natural encoding $\theta(O)=w=w_{1} w_{2} \cdots w_{n} \in \mathcal{W}(n)$ defined by $w_{i}=1$ if $O_{i}$ is obtained from $O_{i-1}$ by adding a square, and $w_{i}=0$ otherwise. Note that palindromic means that $\left(O_{0}, O_{1}, \ldots, O_{n}\right)$ already carries all information of $O$.

Next we conclude the proposition by constructing a bijection $\eta$ from the set of palindromic vacillating tableaux of length $n$ and height bounded by 1 to $\mathcal{W}(n)$. Given such a tableau $V=\left(V_{0}, V_{1}, \ldots, V_{2 n}\right)$, we define $\eta(V)=w=w_{1} w_{2} \cdots w_{n}$ according to the four cases: (i) if $i$ is odd and $V_{i}=V_{i-1}$, then $w_{i}=1$; (ii) if $i$ is even and $V_{i}$ is obtained from $V_{i-1}$ by adding a square, then $w_{i}=1$; (iii) if $i$ is even and $V_{i}=V_{i-1}$, then $w_{i}=0$; (iv) if $i$ is odd and $V_{i}$ is obtained from $V_{i-1}$ by deleting a square, then $w_{i}=0$. Clearly $V$ is a vacillating tableaux if and only if the number of type (ii) moves is no less than the number of type (iv) moves in any initial segment of $V$. This is the same as that in any initial segment $w^{\prime}$ of $w$, even $\left(w^{\prime}, 1\right) \geq \operatorname{odd}\left(w^{\prime}, 0\right)$, which is equivalent to $w \in \mathcal{W}(n)$ by Lemma 11 . Thus $\eta$ is the desired bijection. 


\section{References}

[1] M. Bousquet-Mélou, Four classes of pattern-avoiding permutations under one roof: generating trees with two labels, Electron. J. Combin., 9 (2003), R19.

[2] M. Bousquet-Mélou, Walks in the quarter plane: Kreweras' algebraic model, Ann. Appl. Probab., 15 (2005), 1451-1491.

[3] M. Bousquet-Mélou and G. Xin, On partitions avoiding 3-crossings, Sém. Lothar. Combin., 54 (2005), Art. B54e.

[4] W. Y. C. Chen, E. Y. P. Deng, R. R. X. Du, R. Stanley and C. H. Yan, Crossings and nestings of matchings and partitions, Trans. Amer. Math. Soc., 359 (2007), 1555-1575.

[5] S. V. Fomin, Generalized Robinson-Schensted-Knuth correspondence, J. Soviet Math., 41 (1988), 979-991.

[6] D. E. Knuth, Permutations, matrices and generalized Young tableaux, Pacic J. Math., 34 (1970), 709-727.

[7] C. Krattenthaler, Growth diagrams, and increasing and decreasing chains in fillings of Ferrers shapes, Adv. in Appl. Math., 37 (2006), 404-431.

[8] L. Lipshitz, D-finite power series. J. Algebra, 122 (1989), 353-373.

[9] M. Petkovšek, H. S. Wilf and D. Zeilberger, $A=B$. A K Peters Ltd., Wellesley, MA, 1996.

[10] T. W. Roby, Applications and Extensions of Fomin's Generalization of the Robinson-Schensted Correspondence to Differential Posets, PhD thesis, MIT, Cambridge, MA, 1991.

[11] M. P. Schützenberger, La correspondance de Robinson, in "Combinatoire et Représentation du Groupe Symétrique”, pp. 59-113. Lecture Notes in Math., Vol. 579, Springer, Berlin, 1977.

[12] N. J. A. Sloane, The On-Line Encyclopedia of Integer Sequences, published electronically at www.research.att.com/-njas/sequences.

[13] R. P. Stanley, Enumerative Combinatorics, vol. 2, Cambridge University Press, Cambridge, 1999.

[14] G. Xin, A fast algorithm for MacMahon's partition analysis, Electron. J. Combin., 11 (2004) R58.

[15] G. Xin, A residue theorem for Malcev-Neumann series, Adv. in Appl. Math., 35 (2005), 271-293.

[16] G. Xin, Determinant Formulas Relating to Tableaux of Bounded Height, Adv. in Appl. Math., to appear, Preprint available at arXiv:0704.3381. 